\title{
Approaches to Improve Teachers' Use of Groupware
}

\section{Stefan Bitzer*}

Chair of Application Systems and E-Business

University of Goettingen, Germany

E-mail: sbitzer@uni-goettingen.de

\section{Marco Klein}

Chair of Application Systems and E-Business

University of Goettingen, Germany

E-mail: mklein@uni-goettingen.de

\section{Matthias Schumann}

Chair of Application Systems and E-Business

University of Goettingen, Germany

E-mail: mschuma1@uni-goettingen.de

*Corresponding author

\begin{abstract}
Teachers are confronted with groupware for various reasons. However, the teachers' acceptance of many systems is afflicted. This paper identifies reasons for the refusal and presents approaches to improve the usage of groupware for teachers. On the basis of an empirical survey on an interschool groupware, we discover shortcomings of groupware and corresponding general conditions. In this context we focus on the offered utility and the embedding of groupware in schools. The results of the study show that the motivation of teachers to employ groupware can be improved by implementing specific organizational terms of use and by focussing the utility on document management. Nevertheless, adjustments in both mentioned directions have to be implemented with caution, since they exhibit critical points as well.
\end{abstract}

Keywords: Inter-School Groupware; Empirical Survey; Utility; Organizational Terms of Use

Biographical notes: Stefan Bitzer is a research associate at the Chair of Application Systems and E-Business. Since 2006 he is part of the work group "Knowledge and Education Management". In cooperation with industry partners such as Volkswagen and Siemens Energy he currently explores on knowledge management with Web 2.0.

Marco Klein is a research associate at the Chair of Application Systems and EBusiness, University of Goettingen. Since 2009 he is part of the work group "Knowledge and Education Management". Currently he researches on ITsupport of Human Resource Management and social software in HRMprocesses of large enterprises.

Prof. Dr. Matthias Schumann is a professor at the University of Goettingen since 1991. He is the managing director of the Institute of Information Systems 
and holds the Chair of Information Systems and E-Business. He has (co-) authored numerous books and articles in the area of information systems.

\section{Introduction}

Groupware-platforms for teachers in intra- and inter-school scenarios are assigned to improve the cooperation among teachers (Kirschner \& Wopereis, 2004M Liu, Laffey, \& Cox, 2008) and to facilitate the administration and coordination of important information and processes (Penichet, Marin, Gallud, Lozano, \& Tesoriero, 2007). However, many systems find little acceptance as handling is cumbersome, functions are not according to needs and the respective technology is not perfected (Jiang, Zhang, Li, \& Shi, 2005). Furthermore, the willingness to use these systems is undermined by inefficient organizational terms of use and the establishment of Web 2.0 applications in the private sector, which often integrate functions similar to groupware and moreover are easy to use (Koch, 2008). Thus, the original aim of groupware-platforms of increasing the efficiency of teachers, especially in cooperative and administrative areas, is threatened.

The following paper therefore analyzes possibilities to improve groupware used in the context of schools in order to increase the readiness of teachers to use it. For this purpose, we consider two dimensions - the organizational and the technical. On the one hand we examine the intra-school organizational terms of use and on the other hand we analyze the offered utility, as, until now, these topics were not examined in the context of groupware platforms for teachers. While the arrangement of intra-school organizational terms of use for groupware usage is seen as a possibility to increase the extrinsic willingness to use groupware, the utility is seen as an approach to increase intrinsic motivation. The improvement approaches are based on the presented survey including questionnaires and expert interviews. In this survey, users of a groupware-platform introduced in vocational schools in Lower Saxony were asked about their attitude towards the platform.

This article begins with a presentation of groupware-platforms in schools, with special focus on the BBS-BSCW-groupware-server representing the research subject of the survey (BBS is the abbreviation for vocational schools in Germany, BSCW stands for Basic Support for Cooperative Work). Subsequently the research design of the study is elucidated, in order to de-fine the methodical background of this paper. The following section deals with the results and implications for the examined BBS-BSCW-groupwareserver. Finally, approaches to improve groupware-platforms in general are derived from the perspectives intra-school organizational terms of use and utility. The article closes with a conclusion and an outlook at future developments in the field of groupwareplatforms for teachers.

\section{Groupware in School Education}

As an introduction, we start by classifying Groupware into the superordinate research field of "Computer Supported Collaborative Work (CSCW)". Subsequently, the usage of groupware by teachers is examined. Both things clarify the context of groupwareplatforms in this article. At the end of this section, the BBS-BSCW-groupware-server is presented. 


\subsection{Groupware in the context of $\mathrm{CSCW}$}

Since the beginning of the 1980s, the interdisciplinary research area of computersupported or computerised group work, also referred to as "Computer Supported Cooperative Work" or as "Computer Supported Collaborative Work" (CSCW), has been studied (Lehtinen, Hakkarainen, Lipponen, Rahikainen, \& Muukkonen, 2001). In the context of research in CSCW, one examines configuration, implementation and evaluation of technical systems supporting social interactions (Gross \& Koch 2007). Thus, research analyzes the way that people work together and how this process can be supported by information and communication technology (Stahl, 2004).

The software supporting group work is referred to as groupware (also collaborative software) (Ellis, Gibbs, \& Rein, 1991). Even today, the exact relation between groupware and CSCW is still under vivid discussion. On the one hand, these terms are seen as synonyms (Koch, 2007). On the other hand, groupware is regarded as the practical implementation of knowledge gained through CSCW in an information and communication system (Wainer \& Barsottini, 2008). This article follows the second point of view, as it is widely supported throughout the literature (Bhatt, Gupta, \& Kitchens, 2005) and as it is distinct from other similar terms.

\subsection{Use of Groupware for Teachers}

Nowadays, teachers are dependent on the qualified handling of groupware for the following reasons (see figure 1).

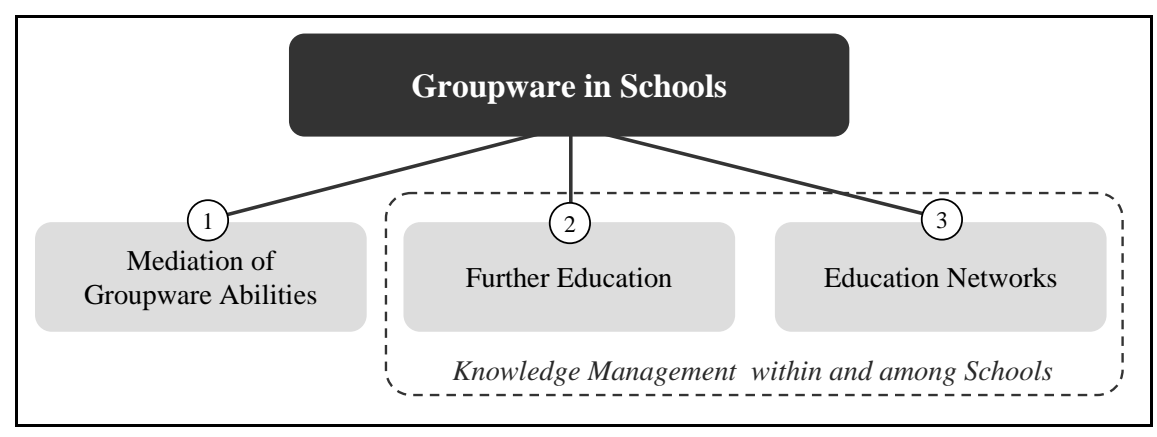

Figure 1. Reasons for groupware use by teachers

\section{Mediation of Groupware Abilities}

Current labour market structures and professions demand a high level of teamwork and media competence, requiring the imparting of such competences in vocational training (Layard, Nickell, \& Jackman, 2005). At the same time, nearly all business branches use groupware (Borghoff \& Schlichter, 2000), so that knowledge and skills on how to use these tools are key qualifications for professional success. However, these qualifications can only be taught if the teacher is acquainted with the respective tools and uses them (Kirschner \& Wopereis, 2003).

\section{Use of Groupware in Further Education}

Using groupware in further training programmes enables the central allocation of teaching materials. Thus, these can be distributed quickly and independent of time and 
place (Bates, 2005). As access is ensured in the long run, materials can be efficiently updated and accessed by all teachers.

\section{Use of Groupware within Education Networks}

A third reason is the set-up of so-called education and qualification networks. An education network consists of several institutions, persons and possibly companies concerned with education and linked e. g. by political or knowledge exchange, friendship or information technical connection (Zgaga, 2003). The use of groupware in education networks does not only enable exchange but also discourse on teaching material between teachers of different locations. This can lead to an improvement of classroom conditions (Zhou, Chen, \& Jin, 2009). Accordingly, these networks play a key role in the continuous qualification of teachers and the improvement of teaching material (Morgan \& Lydon, 2009).

\section{Knowledge Management within and among Schools}

In summary, it can be said that the use of groupware is important for teachers and that it can improve work organisation and the quality of teaching and learning. Additionally, a groupware-platform should also aim at bringing together people, places and content, thus creating a basis for knowledge management within and among schools. Knowledge management includes all actions and tasks that optimise knowledge handling. This is not only essential for businesses in operational knowledge management, but for schools as well (Caroll et al., 2003).

\subsection{BBS-BSCW-Groupware-server as subject of the empirical study}

In 1998, a groupware for teachers was implemented in a project between the Lower Saxony Ministry of Culture and Education and the Institute of Business Information Systems at the Georg-August-University Goettingen in Germany. One of the main goals of this project was to test a communication platform for teachers in the area of informatics at the BBS (abbreviation for vocational schools in Germany). The groupware BSCW (Basic Support for Cooperative Work) was used as software and during the testing period it was administered by the Institute of Business Information Systems. Since 2000, the BBS-BSCW-platform is operated over the Lower Saxony education server (http://bbs-bscw.nibis.de). The software was continuously updated with the available releases. While in the beginning the number of users increased continuously, the augmentation of users stagnated in the last three years (More information on user numbers and user composition is given in section 3.3). To find out reasons for the stagnation and to identify potentials of improvement of the platform, an empirical study was conducted.

The BSCW-system is a web-based document management and communication platform for distributed work on inter- and intranet. Licenses for teaching purposes in schools were provided free of charge. The requirements are a server with operating software and an IP address. Unlike former groupware solutions, such as Lotus Notes and Microsoft Exchange, the BSCW-system is browser-based and thus independent of system software (OrbiTeam Software, 2008). The following table depicts the most important functions of BBS-BSCW-groupware-servers (see table 1), that correspond to the classical functions of a groupware (Penichet, Marin, Gallud, Lozano, \& Tesoriero, 2007). 
Table 1. BBS-BSCW-groupware-platform functions

\begin{tabular}{ll} 
Function & Description \\
\hline \hline Document management & $\begin{array}{l}\text { Organized workgroups are able to locally deposit, edit and } \\
\text { exchange documents in a common work area with the help } \\
\text { of so-called Shared Workspaces. A version management, e- } \\
\text { mail notification in case of changes and blocking mechanism } \\
\text { are available for document management. }\end{array}$ \\
\hline Work area & $\begin{array}{l}\text { Protected work areas can be developed e. g. for further } \\
\text { education. These areas can only be accessed by authorized } \\
\text { users. However, it is also possible to anonymously access } \\
\text { public released content. }\end{array}$ \\
\hline User administration & $\begin{array}{l}\text { The construction of different user groups is enabled by the } \\
\text { administration of user privileges, which also defines their } \\
\text { roles and distributes access authorization (OrbiTeam } \\
\text { Software, 2008). New users can be invited via e-mail. }\end{array}$ \\
\hline Personal resources & $\begin{array}{l}\text { The platform controls the administration of dates, contacts, } \\
\text { tasks and notes. }\end{array}$ \\
\hline Asynchronous & $\begin{array}{l}\text { Asynchronous communication is possible via e-mail service, } \\
\text { discussion forums or notice boards. }\end{array}$ \\
\hline
\end{tabular}

\section{Research Design and Methodology}

To identify improvement factors for groupware used by teachers in the context of vocational and general schools, an empirical study containing questionnaires and interviews and using the BBS-BSCW-Groupware-server as research context was arranged. After a systemization of the current state of research and the definition of the research gaps, the study will be characterised by presenting the research goals and methodology, the sampling and finally restrictions of the survey.

\subsection{Current State of Research}

Even though groupware-platforms for teachers have been used in vocational and general schools for many years, there are only few publications concerning the improvement of teachers' use of respective platforms. Such improvement can result from an idealistic design of groupware and from organizational terms of use, both identified as important factors for groupware usage (Mark, 2002; Ackerman, 2000; Morris \& Dillon, 1996).

The idealistic design can be understood as the optimal combination of usability and utility to provide task-efficiency to their users (Grudin, 1992). Regarding this, only the concept of usability is already examined. Findings from usability surveys of analogous collaborative systems, e. g. Enterprise 2.0 implementations (Hart, Ridley, Taher, Sas, \& Dix, 2008; Doinea \& Van Osch, 2010), can be adapted to groupwareplatforms for teachers, as they base upon comparable usage aims and usage cases (Koch, 2008). 
From the perspective of utility, entailing all functions of software that are requested by users (Nielsen, 2003), insights into platform requirements originate only from specialized perspectives. Theses findings cannot be adapted to groupware-platforms for teacher-teacher interactions. On the one hand, utility findings and ideal functionalities are independent of user-roles (e.g. Ellis, Gibbs, \& Rein, 1991; Gutwin, Roseman, \& Greenberg, 1996; Christiansson, 2001) and therefore are not relevant for the observed teacher-perspective. On the other hand, research is limited to information exchange among students and teachers (Christiansson, 2000; González, García de la Santa, Gorghiu, \& Gorghiu, 2005; Glava, 2007) or to groupware-related cooperation in companies (Ruth, Lorz, \& Braun, 2005), but not to teacher-teacher interactions.

In addition to usability and utility, organizational terms of use can be seen as an important factor for an efficient usage of such platforms (Mark, 2002; Ackerman, 2000; Morris \& Dillon, 1996). The concept of organizational terms of use - in this survey applied as intra-school terms of use - can be understood as opportunities and conventions of organizations to influence the intention of teachers to use software and thus to arrange its usage. Till now it is not examined, how this aspect can be arranged with regard to improve teachers' use of Groupware.

An overview on more fields of empirical research concerning groupwareplatforms is provided by Wainer and Barsottini (2004). They discuss literature on groupware-platforms from a design and a behavioural science approach.

\subsection{Research Goals and Methodical Approach}

As the current state of research on the design of groupware-platforms shows, teachers' opinions on how to improve groupware design and its usage have not yet been investigated - especially in the context of utility and organizational terms of use. However, as teachers show a tendency to working individually rather than team-oriented (Smyth, Dow, Hattam, Reid, \& Shacklock, 2000), groupware should be designed according to user needs, so that usage-readiness and thus also the willingness to work in teams and to actively exchange information will be increased (Liu, Laffey, \& Cox, 2008).

On the background of this research gap, an empirical study focusing on the BBSBSCW-groupware-platform was carried out, to collect qualitative indications of how this platform, as an isolated IT-artifact, can be improved. According to this, the aim of the survey is to find mismatches when using the platform and - building on these - to recommend approaches to improve the BBS-BSCW-server. To examine these approaches, the users of the platform were asked to state their opinions concerning firstly organizational terms of use and secondly the utility and functionality of the platform. This focus ensures a concentration on the identified research gaps, which were identified to be significant for the practical success of a groupware-platform (Mark, 2002; Ackerman, 2000). Thus, the survey provides explicit contribution to theory and practice.

Within the survey, which was carried out in winter 2008, the user opinions were collected through qualitative expert interviews with teachers and a quantitative online questionnaire on the presented groupware-platform. The questionnaires were put online on the groupware-platform for one month, so that it could be filled out voluntarily and anonymous. Additionally, all platform-users were informed about the survey by e-mail. Items with alternative answers and open answer possibilities were included, all based on nominal scales. This is supposed to support the study's inductive character. Assessment questions arranged on an ordinal scale are an exception. 
The semi-structured expert interviews basically provide a range of qualitative opinions on how to improve the organizational terms of use and the utility, so the interview guideline was geared to the content of the questionnaires-items. Time-related, they were conducted after the questionnaires, in the form of one-on-one interviews with platform users in their vocational schools. The interview participants were picked randomly. Findings and impressions of the filled questionnaires were not used for interviewing the experts, to provide unaffected opinions relating to the interviewer and the interviewee. The answers were digitally recorded and later transliterated into a textform.

The research design is not experimental, as differentiating between control and test group is not methodically sensible in this inductive research project. In addition, experimental grouping would not add any value. Instead, it is the explicit goal to examine the present motivation, typical usage scenarios and the present opinions on utility. The findings base on previous usage experiences of the sample as one homogenous collective. Statistical evaluation is done descriptively, according to the inductive and investigative research methodology. Multi-variant methods are thus not applied.

\subsection{Sampling}

All of the teachers interviewed during the study are users of the BBS-BSCW-groupwareplatform and teach at a vocational school in Lower Saxony, Germany. Therefore, the sample population consists of all users of this platform teaching in this geographical area, which amounts to 4,850 users at present. Since accounts are deleted after one year without login, there are no dead user accounts. As Lower Saxony has about 9,700 vocational school teachers, with about 640 trainee teachers, only every second teacher is registered on the BBS-BSCW-server (Lower Saxony Ministry of Culture and Education, 2007). This discrepancy between the number of active school teachers and the number of platform-users shows that this user group can be increased in the future, which emphasises the potential of the BBS-BSCW-groupware-server to improve.

The selection of the questionnaire sample was random, in order to achieve a large sample size. Finally 253 teachers filled out the questionnaire, so the response rate with regard to 4,850 users was approximately $5 \%$. The distribution between the genders was not evenly distributed, as $60 \%$ of the participants were male and $38 \%$ were female while $2 \%$ did not state their gender. However, this uneven distribution roughly corresponds to the sex distribution of teachers in Lower Saxony (Lower Saxony Ministry of Culture and Education, 2007) and of all platform users. Sample ages homogeneously distributed among the birth years of 1950-1959, 1960-1969 und 1970-1979 (see figure 2).

The age-groups correspond to the age distribution of the current platform-users. Compared to the age distribution of vocational school teachers in Lower Saxony (Lower Saxony Ministry of Culture and Education, 2007), especially older teachers born between 1940 and 1949 did not took part in the study and furthermore do not use the platform. This limitation has to be considered when generalising the results.

The sample consisted of $75 \%$ full-time teachers, $22 \%$ trainee teachers and $3 \%$ staff with other functions. Even though this also corresponds to the distribution to the platform users and thus of the population, it does not correspond to all teachers. In this case, trainees make up 5\% of all teachers (Ministry of Culture of Lower Saxony, 2007).

This shows that more trainees use the platform and took part in the survey than full-time teachers. 


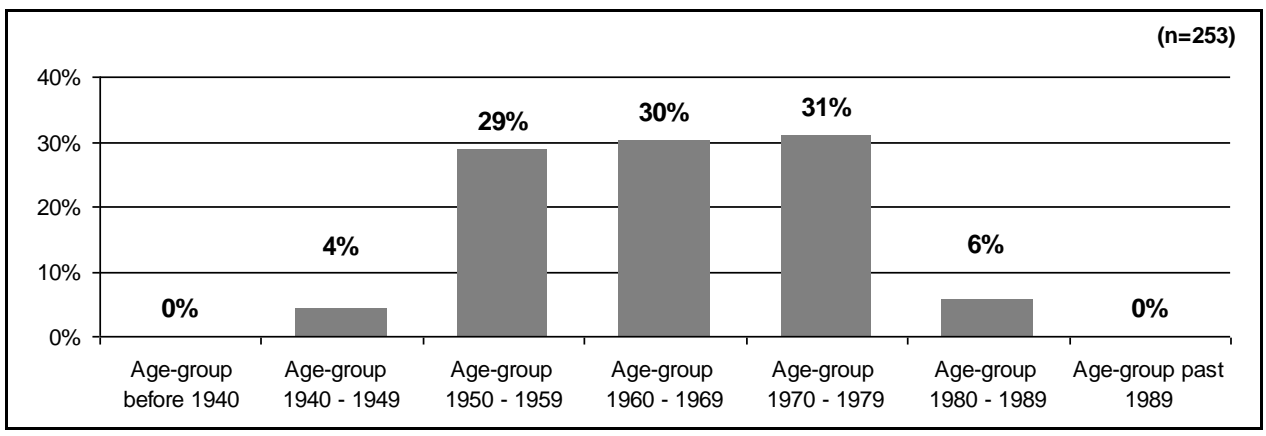

Figure 2. Sampling of the questionnaire - age distribution

As the expert interviews focus on qualitative aspects, a small sample size of twelve participants, who were picked randomly, was chosen for the discussions. None of the participants has filled out the questionnaires, so the interview-findings can be seen as supplemental findings to the quantitative data. All experts were employed as full-time teachers. Ten participants were male and two female. The age distribution corresponds to the questionnaire sample.

\subsection{Restrictions and limitations of the study}

The study is subjected to restrictions, which were considered when examining and analyzing the findings. First of all, the survey provides merely findings from one practical case of groupware-platforms for teachers, so this sample is hardly representative (Stake, 1995). However, as the examined platform provides standard groupwarefunctionality (see section 2.3), the approaches to improve groupware can be generalized to all platforms matching the standard-functions proposed by Penichet, Marin, Gallud, Lozano, and Tesoriero (2007).

Another limitation can be derived from the participants in the study. On the one hand, the sampling-information (see section 3.3) shows that the results can be generally applied to all platform users. On the other hand this does not hold true for all teachers in Lower Saxony at vocational schools, as the sample deviates with regard to age and role distribution. In general, correlations between the discovered mismatches and the developed approaches to reduce them have to be validated using firstly more cases and secondly quantitative research methods, e.g. structural equation models (Anderson \& Gerbing, 1988). For these, the findings of the BBS-BSCW case provide the corresponding backgrounds and hypotheses. Thus, from a statistical point of view, the validity of the findings cannot be guaranteed. The same applies for the statistical reliability of the constructs, which were measured with single items. Then again, the constructs asked in the questionnaires, were supported by questions in the semi-structured expert interviews, referring to the questionnaire's items. Consequently, the causal reliability is assured.

Furthermore, as the study examined a platform at vocational schools, it has to be investigated whether the results are also applicable to teachers and groupware-platforms of schools in general. Another restriction is the fact that students were not asked for their opinion on groupware-solutions. Thus, approaches on how to improve groupware for exchanging information and documents between students and teachers are not part of the findings, but can be found in other empirical surveys (e. g. Christiansson, 2000; González, García de la Santa, Gorghiu, \& Gorghiu, 2005; Glava, 2007). 


\section{Research Results and Implications regarding Organizational terms of use}

\subsection{Findings}

First, we analyze usage frequency and duration. All in all, the usage frequency is moderate (Emmanouilides \& Hammond, 2000): 24\% of the participants use the BBSBSCW-platform more than once a week, $61 \%$ at least once a month. Usage duration is rather short. The moderate but constant usage frequency in combination with the high share in the target population allows us to infer that the groupware is, at least to some extent, necessary for teachers for reasons discussed later in this section. Nevertheless, this does not reveal anything about the satisfaction of the users regarding the platform. Regular and short usage can also be noticed in the use of platform functionalities (see section 4.2) and was confirmed in the interviews.

The analysis of the motivation for usage (figure 3), in connection with the usage frequency and duration, is the basis for the analysis of groupware improvement (Pipka, 2004). The results show that the groupware is mostly used for group work within schools $(51 \%)$. Furthermore, the use of the platform for teacher training seminars $(18 \%)$ corresponds to the number of participating trainees $(22 \%)$ so that an increase in this area can hardly be expected. However, the use of groupware to support cooperation within schools shows a great potential for development, as each participant in the survey is both a platform user and a teacher at a school. Thus, all participants could use the groupware to cooperate inside the schools without great effort. Even though it is reasonable to believe that not every teacher wants to cooperate within his/her school, the usage motivation in this area could be increased significantly. In addition, cooperation among teachers within a school is considered to be especially important for the development of a school (Hofman, Hofman, \& Guldemond, 2001).

On the basis of the described study results, we discuss potential measures to improve the usage motivation and to provide additional motives in the expert interviews. In the context of teachers, different levels of directives issued by the school are the most obvious solution. An obligation to use BBS-BSCW-groupware-servers as the strongest type of directive was seen as counterproductive. On the one hand, the platform would take on a more official character and usage would therefore increase. On the other hand, however, four out of twelve participants did not think that this was feasible due to organizational limitations. Furthermore, especially older teachers would resent this action and their motivation to use the servers would sink. The interview partners therefore think that a usage recommendation would be more adequate. Additionally, teachers could also be encouraged to use the servers by an indirect order, for example by distributing curricula or notice boards exclusively via the platform.

Increasing the cooperation among schools proves to be more difficult. Thus, all participants think that a general directive to use the servers does not make any sense, as this would contradict the schools' autonomy. School autonomy, as an institutional fact in Germany, is seen as important and as increasing efficiency (Woesmann, 2007). According to the study results, cooperation among schools via groupware-platforms can only be increased by incentives, such as the indirect usage directives mentioned above. In summary, usage directives are a challenge for the use of groupware within and among schools. 


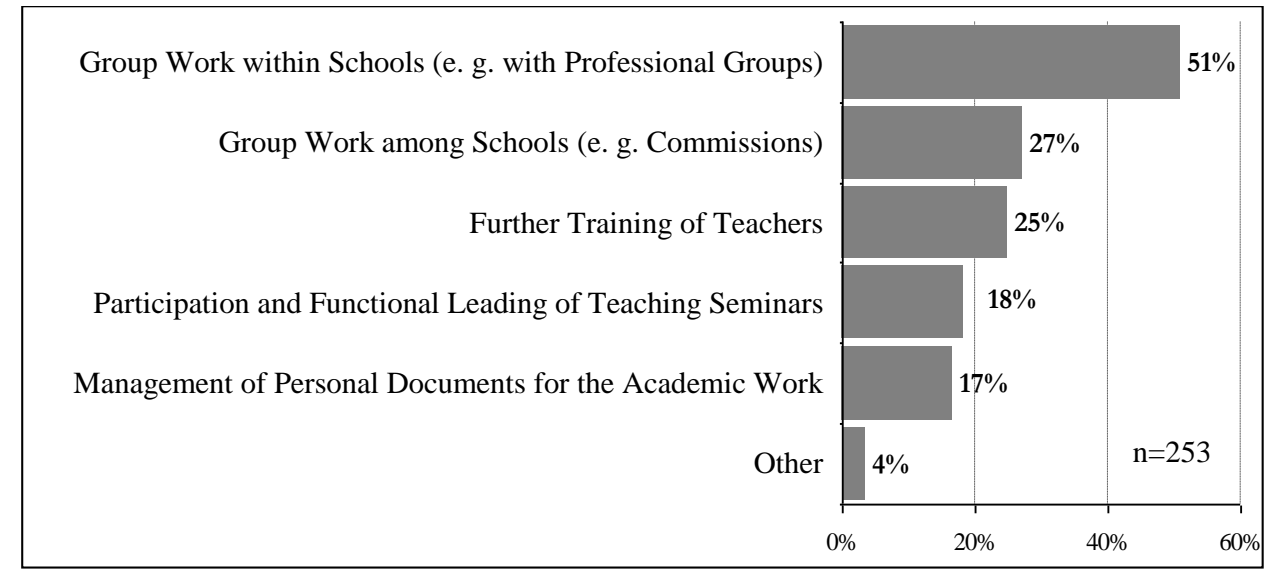

Figure 3. Study results - usage motivation

Having discussed the general use of the platform with its different application areas, the next step will be to examine reasons why only a small amount of material is uploaded. This can arise by reason of technical limitations of the platform, or else by organizational problems. For this purpose, the participants were asked in the interviews which reasons prevented them from posting material to the platform. Here, typical problems connected to groupware and knowledge sharing could be observed (Azudin, Ismail, \& Taherali, 2009). Even though the reasons may be manifold, they all lead to a low distribution of material and an imbalance between "give and take". The importance of the latter for a lasting and successful employment of groupware in the learning environment has already been stated by others (Kreijns, Kirschner, \& Jochems, 2003). According to the expert interviews, the reasons against posting material are mostly of a psychological nature and therefore belong to the organizational aspects. They are listed below according to relevance in the interviews. The relevance was corresponding to frequency of mention:

1. insecurity, fear of being criticised

2. lone-fighter-syndrome

3. technical problems with the platform

4. lack of time due to high workload

The highest barrier for teachers is the insecurity concerning the quality of their work and fear of being criticized. In this context, the teachers' "lone-fighter-mentality" is a handicap. Especially in Germany, it has been identified as a great barrier for cooperation among teachers in many areas (Herrmann, 2007). Even though high workloads, technical difficulties and other reasons were mentioned, they are not crucial and will not be elaborated in this paper any further. Thus, another important challenge can be found in the mental barriers described above. 


\subsection{Approaches to improvement}

The success and utilization of groupware for teachers, just like comparable business software, depends highly on the organizational terms of use (Mark \& Poltrock, 2001; Hong \& Kim, 2002). We identified in our case two interdependent challenges:

1. Design of directives or recommendations for the platform utilisation

2. Dealing with mental barriers

Directives or recommendations on how to use the groupware have to be designed sensibly. The indirect usage instructions via exclusive information and content distribution through the platform (see section 4.1) have already been successfully implemented by the user group of trainees. The exclusive distribution of, for example, information for study seminars over the BSCW-server, has increased the share of trainees using the platform to close to $100 \%$. Especially for the easy and at the same time important increased utilisation of the groupware within schools (see section 4.1), indirect instructions, like the distribution of substitution schedules over the platform, possess high potential. However, these usage recommendations should be given by each school separately in order to respect their autonomy.

The second challenge, breaking down mental barriers, is a difficult and continuous process. This process can be supported by organizational as well as technical measures (see section 5.2). To reduce the insecurity and the fear of being criticised, one should explicitly refer all participants of the platform to obey the netiquette (Shea, 1994). The netiquette is a set of social conventions that should lead to polite communication between the users. These rules of conduct could even be enhanced regarding commendations for teachers that actively participate on the platform in a positive way. The lone-fighter-mentality can be counteracted with rewards for participation. Even though in the long run users should benefit from the software system itself, in the beginning incentives can help to increase the attendance (Vassileva, 2003). In our context, financial incentives are probably neither available nor reasonable, especially in voluntary inter-school networks. However, commendations coming from important persons, such as principals or project leaders, can be used instead and effect the motivation.

\section{Research Results and Implications regarding utility}

\subsection{Findings}

In the first step, we analyzed the functions being currently used compared to the functions teachers wish for. The values are very similar, with a few exceptions. That allowed us to draw the following conclusions:

1. Functions often required are also used frequently. Seen the other way round, functions not required often are also not used.

2. The required functions are at least available in a satisfactory form, as they would otherwise be required but not used. 
The results show (see figure 4) that the BBS-BSCW-server is primarily used for the posting and editing of documents, as well as for the distribution of official information.

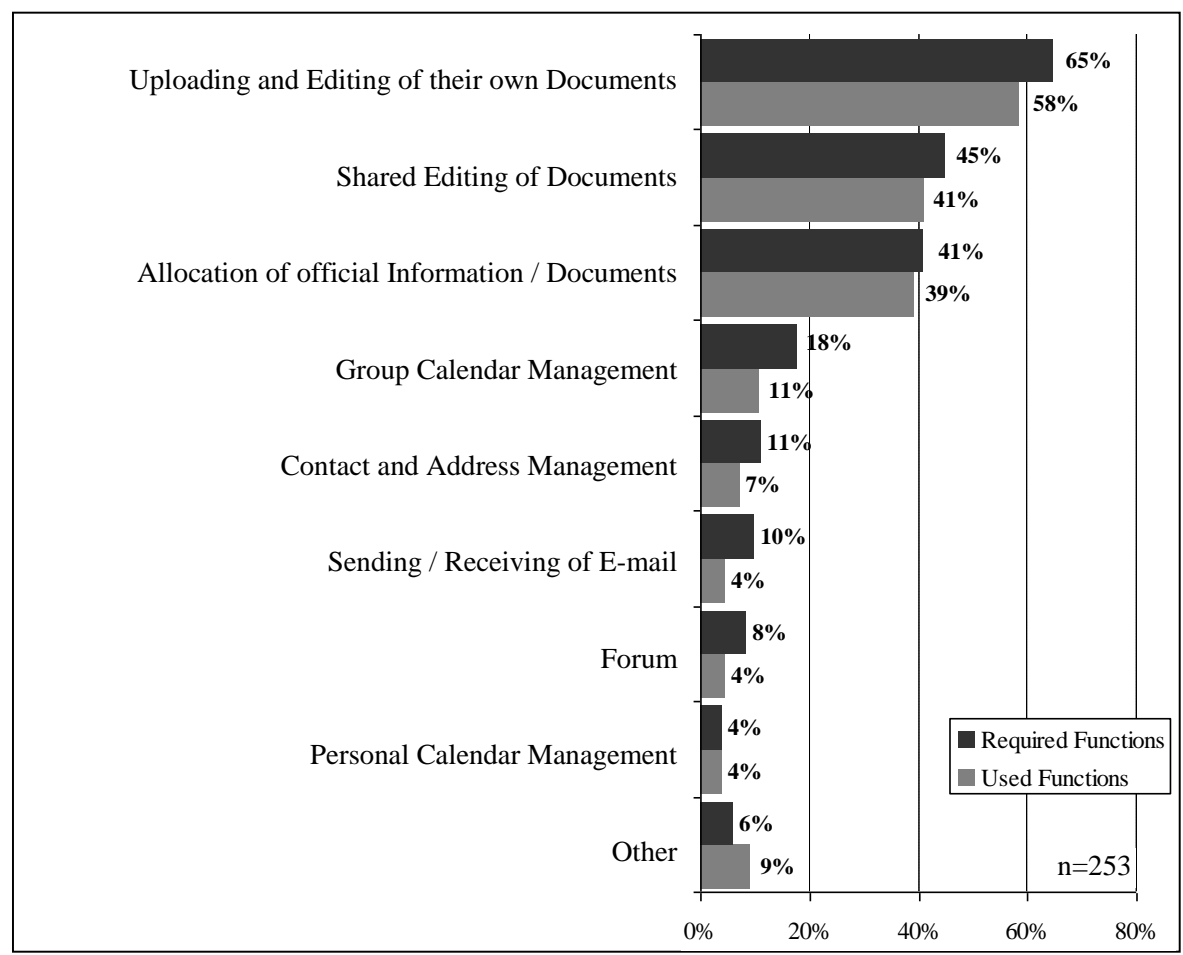

Figure 4. Study results - functions used and required

The actual editing of documents is done locally, whereas the BSCW functions as a central storage with version management and lock mechanisms. This is due to the fact that the platform does not support central, synchronic editing of documents. In addition, $18 \%$ of the participants requested the administration of group appointments, but only $11 \%$ actually used this function. The current implementation of this function does not seem to be sufficient. In the areas of contact management, integrated e-mail function and the forum, the request for these functions is also higher than their utilisation. However, the difference is not significant. Only every tenth person asked for one of these functions. At the same time their utilization frequency is even lower. The results concerning the functions required and actually used were emphasised by the expert interviews. The platform was nearly exclusively used for data storage and exchange of material. Administration of e-mail, contacts and appointments was neither required nor used.

The functions described explain the results stated in section 4.1 of frequent but short logins. Posting and downloading documents only takes a short time, whereas time consuming functions, such as emails, activities in forums or management of appointments were rarely used. This trend can also be observed for the required additional functions. Here, especially complementary functions for document management are named. $21 \%$ of the participants ask for a data synchronisation and $20 \%$ wish to be able to work on the same document simultaneously (see figure 5). $21 \%$ also think that supporting the workflow management of the school's administration processes 
will improve the platform. The participants saw further improvement potential in the management of students' achievements and the synchronisation of appointments.

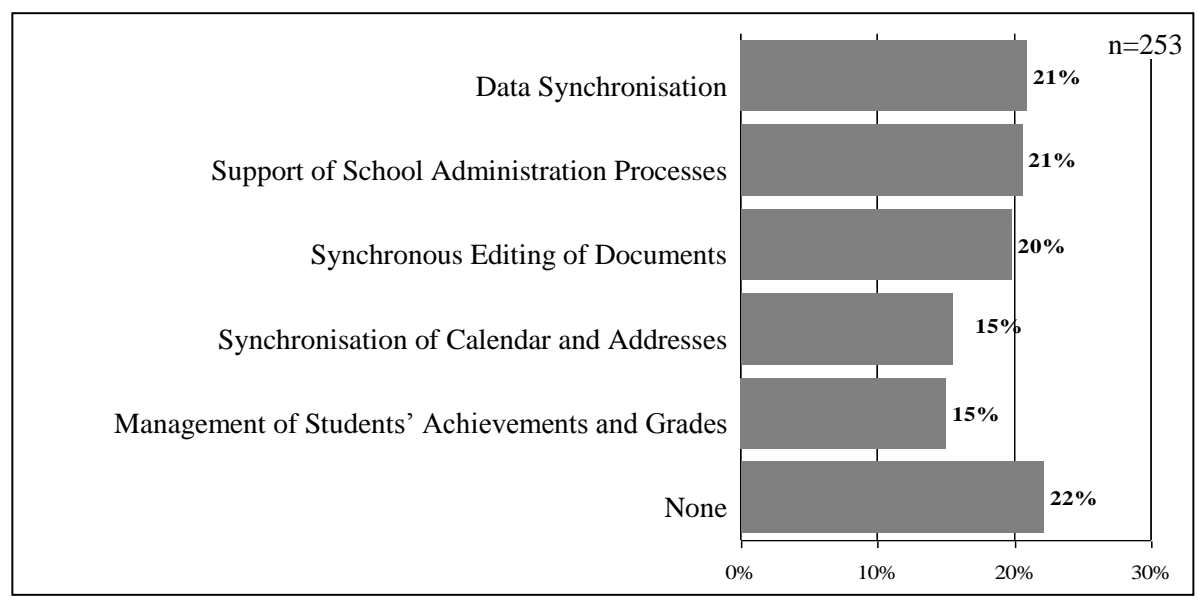

\section{Figure 5. Study results - possible extra platform functions}

The expert interviews also showed document management as a central improvement factor. An easier exchange of documents and mechanisms to set access rights not only for workspaces but for documents and folders were seen as important. Additionally, the possibility of anonymously uploading content was seen as beneficial. This suggestion could counter the teacher's insecurity and fear of criticism, as mentioned in section 4 .

\subsection{Approaches to Improvement}

The study shows that in order to increase the acceptance and the success of a groupware for teachers, it is not important to offer a broad range of functions. The platform should rather focus on core functions and their adequate support. Other studies in the area of information systems have shown similar results (Kim, 1998). Looking at the investigated functions of the groupware as presented in section 2.3, it becomes clear that most participants concentrate on exchange of data and documents and therefore on document management functions. The utility could be optimized by efficiently embedding a groupware-document management system into local explorer services, for example via WebDAV (Whitehead Jr. \& Wiggins, 1998). In this context, the possibility of acting anonymously on the platform can be discussed as well. This could help reducing the insecurity of teachers regarding their uploaded material. Nevertheless, one have to critically consider that in the long run, a groupware for teachers only makes sense if persons can actively exchange and discuss information. This is hardly possible while remaining anonymous. As a compromise between uploading content anonymously and authored articles, the use of separate work areas could be strengthened in the future, for example, in form of intra-school or small, intra-working group areas. This method can also reduce the mental barrier, like the anonymous uploading of content. However, drawbacks of anonymity are avoided and the possibility of actively exchanging and discussing within the separate areas is preserved. Further studies have to show how far this method can actually reduce mental barriers. In contrast to document management functions, personal resources and possibilities of asynchronous communication are rarely 
requested or used. This makes sense, as these tasks are mainly tackled by using other tools, which are used either privately or officially (e.g. MS Outlook, Thunderbird or Webmailer). Consequently, such tools have to be maintained additionally in the groupware.

In addition to the explained focus on document management, the platform could be enhanced with functions decreasing mental barriers and increasing user participation. As mentioned in the previous section, rewards or incentives are a potential solution. These can be implemented by different types of reputation systems, e. g. rating or ranking solutions (Lytras, Damiani, \& Ordóñez de Pablos, 2008). This allows teachers to build a professional reputation, which increases the motivation to share content and the level of collaboration (Wasko \& Faraj, 2005). Furthermore, users can be stimulated through positive comments and animated by their collecting passion. For example, users could earn digital points for participation via automatic ranking systems. Especially new Web 2.0 approaches, mainly rating systems, promise to increase user participation in the content creation process and solve the presented problems (Adler \& de Alfaro, 2006). However, one has to consider that users can also get critical or negative reviews in rating systems. Keeping in mind the teacher's fear of negative comments (see section 4.1), an implementation of this function can also result in problems.

As we described in Section 2.2, collaboration between teachers is an important part for continuous qualification and improvement of teaching material. This collaboration cannot be achieved by exclusively focusing on document management. Currently, the discussion forum is a separate component. We propose to integrate the discussion and the documents, as it is successfully the case with Wikis (Schwartz, Clark, Cossarin, \& Rudolph, 2004). A discussion has to be directly connected to a work area or a specific document. In this way, discussions not belonging to the respective document or area are blanked out. This improves the clarity of the discussions and, in case of separate work areas, leads to a protected and private communication. Table 2 summarizes the suggested utility of the groupware platform.

\section{Table 2. Proposed functions of the BBS-BSCW-groupware}

\section{Function Description}

Should be enhanced; e. g. by embedding platform into

Document management local explorer services, possibility of participating anonymously.

\begin{tabular}{ll}
\hline Work area & Separate work areas should be strengthened. \\
\hline User administration & No changes. \\
\hline Personal resources & Should be deactivated. \\
\hline $\begin{array}{l}\text { Asynchronous } \\
\text { communication }\end{array}$ & $\begin{array}{l}\text { E-mail service and notice boards should be deactivated; } \\
\text { merging of discussion forums with document } \\
\text { management to improve collaboration. }\end{array}$ \\
\hline Reputation Systems & Ranking and / or rating systems should be implemented. \\
\hline
\end{tabular}




\section{Conclusion and Outlook}

On the basis of this survey, we identified shortcomings of the analyzed BBS-BSCWgroupware-server und suggested approaches to improve the platform. In detail, recommendations for organizational terms of use and the platform utilization were acquired. In matters of organizational terms of use, the design of directives for the platform utilization is an important aspect. Indirect usage instructions allow increasing the use of the platform. As shown, the process of breaking down mental barriers can be supported by organizational and technical measures. The first mentioned measures are mainly based on social behaviour and do not risk negative consequences. However, the implementation of reputation systems, especially rating systems, can potentially backfire on the insecurity and motivation of teachers in case of negative comments. Nevertheless, studies showed a positive effect on collaboration (Wasko \& Faraj, 2005) and communication behaviour (Jøsang, Ismail, \& Boyd, 2007), so we propose to enhance the platform with this kind of feature. In addition, we suggest adapting the utility on the exchange and management of documents, as these are currently the most important functions for teachers. However, it has to be noted that this strong cutback of functions would reduce the platform to a kind of document management system. This objection can be addressed with the described merging of discussion forum and document management. Nevertheless, one has to face the question whether a groupware is the right tool for the described scenario or if a web-based document management system would be a more appropriate solution. Due to the requirements explained in section 2.2, we argue that in our case groupware is the right kind of software. Furthermore, we think that our suggested measures help achieving the actual goal of the platform for teachers: supporting and increasing group work (Ellis, Gibbs, \& Rein, 1991). In Figure 6 we summarize our identified measures to adjust groupware to teachers' needs.

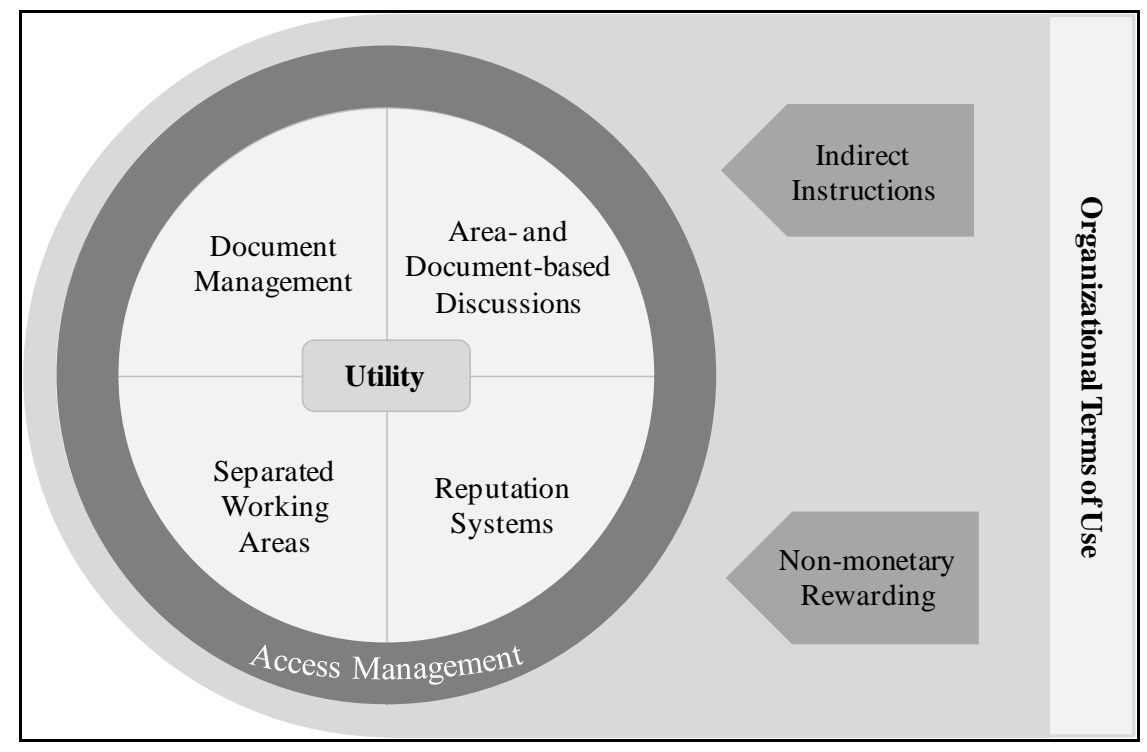

Figure 6. Framework for teacher adjusted groupware 
After our study, some of the suggestions were implemented. The document management functions were enhanced by integrating the platform into local explorer services via WebDAV and improving the functionality of the work spaces. Other measures, like the possibility of participating anonymously and the implementation of reputation systems, are still in progress. However, after an internal discussion, the proposed deactivation of platform functions such as personal resources and e-mail service, were declined. For the final evaluation of the findings, the developed approaches should be validated after all planned realizations are done.

This article shows that from a practical point of view, a broad functional range is not important for a successful usage of groupware for teachers. In fact, the utility should primarily focus on document exchange between teachers. Furthermore, in practice, incentives for use of the platform are needed and inhibitions of teachers have to be reduced. From a theory's perspective we showed that groupware is used and necessary for teachers in school education, but have to be adapted to the respective scenario. In addition, this article reveals different directions for future research for the use of information technology in the field of education. One has to analyze to what extent the implementation of reputation systems has a positive effect on the involved users and if it is applicable to other tools in education. In this process, different educational environments and user groups will probably lead to different results and therefore have to be considered as well. Similar applies for the reduced functionality. Here, too, one has to evaluate the success of this measure, check the transferability of the results and derive important basic conditions and requirements. Finally, there are important questions in context with the described indirect usage instructions. Future research has to clarify, what kind of indirect instructions do exist and to what extent they have negative impacts on users or their contribution.

\section{References}

1. Ackerman, M. S. (2000). The Intellectual Challenge of CSCW: The Gap Between Social Requirements and Technical Feasibility. Human-Computer Interaction, 15(2), 179-203.

2. Adler, B. T., \& de Alfaro, L. (2007). A Content-Driven Reputation System for the Wikipedia. Proceedings of the 16th Intl. World Wide Web Conference, Banf, Canada.

3. Anderson, J. C., \& Gerbing, D. W. (1988). Structural Equation Modeling in Practice: A Review and Recommended Two-Step Approach. Psychological Bulletin, 103(3), $411-423$.

4. Azudin, N., Ismail, M. N., \& Taherali, Z. (2009) Knowledge sharing among workers: a study on their contribution through informal communication in Cyberjaya, Malaysia. Knowledge Management \& E-Learning: An International Journal, 1(2), 139-162.

5. Bates, A. W. (2005). Technology, E-Learning and Distance Education. London: Routledge.

6. Bhatt, G., Gupta, J. N. D., \& Kitchens, F. (2005). An exploratory study of groupware use in the knowledge management process. Journal of Enterprise Information Management, 18(1), 28-46.

7. Borghoff, U. M., \& Schlichter, J. H. (2000). Computer-Support Cooperative Work Introduction to Distributed Systems. Heidelberg: Springer. 
8. Caroll, J. M., Choo, C. W., Dunlap, D. R., Isenhour, P. L., Kerr, S. T., MacLean, A., \& Rosson, M. R. (2003). Knowledge Management Support for Teachers. Educational Technology, Research and Development, 51(4), 42-64.

9. Christiansson, P. (2000). IT in distributed open learning environments. In G. Gudnason (Ed), Construction Information Technology 2000 - Taking the Construction Industry into the 21stcentury (pp. 197-208). Icelandic Building Research Institute.

10. Christiansson, P. (2001). Experiences from using internet based collaboration. Proceedings of the Conference on Architectural Research and Information Technology (pp. 103-112). Aarhus, Netherlands.

11. Doinea, M., \& Van Osch, W. (2010). Collaborative Systems: Defining and Measuring Quality Characteristics. Journal of Applied Collaborative Systems, 2(1), $50-61$.

12. Ellis, C. A., Gibbs, S. J., \& Rein, G. (2001). Groupware: some issues and experiences. Communications of the ACM, 34 (1), 38-58.

13. Emmanouilides, C., \& Hammond, K. (2000). Internet usage: Predictors of active users and frequency of use. Journal of Interactive Marketing, 14 (2), 17-32.

14. Glava, C. (2007). BSCW as a virtual classroom - results of a research based approach regarding the educational features of the platform. Proceedings of ICT in education: reflections and perspectives (pp. 150-157). Bucharest, Hungary.

15. González, V. R., García de la Santa, A., Gorghiu, G., \& Gorghiu, L. M. (2005). BSCW as a support system for distance teacher training. Proceedings of the Third International Conference on Multimedia \& ICT's in Education (pp. 696-701). Caceres, Spain.

16. Goodwin, N. C. (1987). Functionality and usability. Communications of the ACM, 30 (3), 229-233.

17. Gross, T., \& Koch, M. (2007). Computer-supported Cooperative work. Munich: Oldenbourg Publishing.

18. Grudin, J. (1992). Utility and Usability: research issues and development contexts. Interacting with Computers, 4 (2), 209-217.

19. Gutwin, C., Roseman, M., \& Greenberg, S. (1996). A Usability Study of Awareness Widgets in a Shared Workspace groupware System. Proceedings of the 1996 ACM conference on Computer supported cooperative work (pp. 258-267). Boston, USA.

20. Hart, J., Ridley, C., Taher, F., Sas, C., \& Dix, A. (2008). Exploring the Facebook Experience: A New Approach to Usability. Proceedings of the NordiCHI, (pp. 471.474). Lund, Sweden.

21. Herrmann, U. (2007). In der Pädagogik etwas bewegen: Impulse für Bildungspolitik und Schulentwicklung. Basel: Beltz.

22. Hofman, R. H., Hofman, W. H. A., \& Guldemond, H. (2001). The effectiveness of cohesive schools. International Journal of Leadership in Education, 4(2), 115-135.

23. Hong, K., \& Kim, Y. (2002). The critical success factors for ERP implementation: an organizational fit perspective. Information \& Management, 40(1), 25-40. 
24. Jiang, J., Zhang, S., Li, Y., \& Shi, M. (2005). CoFrame: A Framework for CSCW Applications Based on Grid and Web Services. Proceedings of the IEEE International Conference on Web Services (pp. 570-577), Orlando, USA.

25. Jøsang, A., Ismail, R., \& Boyd, C. (2007). A survey of trust and reputation systems for online service provision. Decision Support Systems, 43(1), 618-644.

26. Kim, J. (1998). Hierarchical Structure of Intranet Functions and Their Relative Importance: Using the Analytic Hierarchy Process for Virtual Organizations. Decision Support Systems, 23(1), 59-74.

27. Kirschner, P., \& Wopereis, I. G. J. H. (2003). Mindtools for Teacher Communities: a European perspective. Technology, Pedagogy and Education, 12(1), 105-124.

28. Koch, M. (2008). CSCW and Enterprise 2.0 - towards an Integrated Perspective. Proceedings of the 21th Bled eConference eCollaboration (pp. 416-427). Bled, Slovenia.

29. Kreijns, K., Kirschner, P. A., \& Jochems, W. (2003). Identifying the pitfalls for social interaction in computer-supported collaborative learning environments: a review of the research. Computers in Human Behavior, 19(3), 335-353.

30. Layard, R., Nickell, S., \& Jackman, R. (2005) Unemployment: macroeconomic performance and the labour market. New York: Oxford University Press.

31. Lower Saxony Ministry of Culture and Education (2007). Die niedersächsischen berufsbildenden Schulen in Zahlen, Schuljahr 2007/2008. Retrieved November 3, 2009, from http://cdl.niedersachsen.de/blob/images/C50575689_L20.pdf.

32. Lehtinen, E., Hakkarainen, K., Lipponen, L., Rahikainen, M., \& Muukkonen, H. (2001). Computer supported collaborative learning: A review. CL-Net Project. $\begin{array}{llll}\text { Retrieved November } & \text { 2009, from }\end{array}$ http://www.comlab.hut.fi/opetus/205/etatehtava1.pdf.

33. Liu, P.-J., Laffey, J. M., \& Cox, K. R. (2008). Operationalization of Technology Use and Cooperation in CSCW. Proceedings of ACM Conference on ComputerSupported Cooperative Work 2008 (pp. 505-514). San Diego, USA.

34. Lytras, M. D., Damiani, E., \& Ordóñez de Pablos, P. (2008). Web 2.0: The Business Model. Oviedo, Spain: Springer.

35. Mark, G. (2002). Conventions and Commitments in Distributed CSCW Groups. Computer Supported Cooperative Work, 11 (3-4), 349-387.

36. Mark, G., \& Poltrock, S. (2001). Diffusion of a collaborative technology cross distance. Proceedings of the 2001 International Conference on Supporting Group Work (pp. 232-241). Boulder, Colorado, USA.

37. Morris, M. G., \& Dillon, A. P. (1996). The importance of usability in the establishment of organizational software standards for end user computing. International Journal of Human Computer Studies, 45(2), 243-258.

38. Morgan, B., \& Lydon, J. (2009). Bologna: some thoughts on its effect on the internationalisation of higher education. Journal of applied research in higher education, 1(1), 63-72.

39. OrbiTeam Software (2008). BSCW 4.4 Manual. Retrieved November 3, 2009, from http://www.bscw.de/files/Download/Help/bscw_help_44_de.pdf. 
40. Penichet, V. M. R., Marin, I., Gallud, J.A., Lozano, M. D., \& Tesoriero, R. (2007). A Classification Method for CSCW Systems. Electronic Notes in Theoretical Computer Science, 168, 237-247.

41. Pipka, J. U. (2004). Development Upside Down: Following the Test First Trail. Proceedings of the $18^{\text {th }}$ European Conference on Object Oriented Programming. Oslo, Norway.

42. Ruth, D., Lorz, A., \& Braun, I. (2005). Web-basierte groupware-Anwendungen für die Kooperation in verteilten Projektteams und virtuellen Unternehmen. Proceedings of the Workshop on Virtuelle Organisation und Neue Medien, GeNeMe 2005 (pp. 121-134). Dresden, Germany.

43. Schwartz, L., Clark, S., Cossarin, M., \& Rudolph, J. (2004). Educational Wikis: features and selection criteria. The International Review of Research in Open and Distance Learning, 5(1).

44. Shea, V. (1994). Core Rules of Netiquette. Educom Review, 29(5), 58-62.

45. Smyth, J., Dow, A., Hattam, R., Reid, A., \& Shacklock, G. (2000). Teachers' Work in a Globalizing Economy, New York: Falmer Press.

46. Stahl, G. (2004). Groupware goes to school: adapting BSCW to the classroom. International Journal of Computer Applications in Technology, 19(3), 162-174

47. Stake, R. E. (1995). The art of case study research. Thousand Oaks, CA: Sage.

48. Vassileva, J. (2003). Motivating Participation in Peer to Peer Communities. Lecture Notes in Computer Science, 2577/2003, 18-23.

49. Wainer, J., \& Barsottini, C. (2004). Empirical research in CSCW-a review of the ACM/CSCW conferences from 1998 to 2004. Journal of the Brazilian Computer Society, 13(3), 27-36.

50. Wasko, M. M., \& Faraj, S. (2005). Why should I care? Examining Social Capital and Knowledge Contribution in Electronic Networks of Practics. MIS Quarterly, 29(1), 35-57.

51. Whitehead Jr. E. J., \& Wiggins, M. (1998). WebDAV: IEFT standard for collaborative authoring on the Web. IEEE Internet Computing, 2(5), 34-40.

52. Woesmann, L. (2007). Fundamental Determinants of School Efficiency and Equity: German States as a Microcosm for OECD Countries. IZA Discussion Paper, No. 2880.

53. Zhou, J., Chen, S. \& Jin, L. (2009). Using Digital Resources for the ECE Curriculum in China: Current Needs and Future Development. Knowledge Management \& ELearning: An International Journal, 1(4), 285-294.

54. Zgaga, P. (2003). The Bologna Process between Prague 2001 and Berlin 2003: Contributions to Higher Education Policy. Proceedings of the Conference of European Ministers Responsible for Higher Education. Berlin, Germany. 